\title{
BAJO EL CIELO DEL ANTIPURGATORIO
}

\author{
Under the sky of the anti-purgatory
}

José Blanco *

\section{RESUMEN}

En el viaje poético de Dante por la ultratumba, las referencias astronómicas establecen su recorrido cronológico. Mientras se encuentra bajo el cielo boreal, todo se ajusta a la visión tolemaica. Pero en el hemisferio austral -donde se encuentra la montańa del Purgatorioel poeta debe trabajar sobre todo con alegorías y, por ello, no es aceptable una posible identificación de la Cruz del Sur en esa imaginaria bóveda celeste.

Palabras clave: Dante, astronomía, purgatorio, Cruz del Sur.

\begin{abstract}
During the Dante's poetic journey on the afterworld, the astronomic references set his chronological route. When he is under the boreal sky, everything is adjusted on the Ptolemaic vision. But in the austral hemisphere, where there is the mountain of Purgatory, the poet must work first of all with allegories and, because of that, is not acceptable a possible identification of the Southern Cross in this imaginary vault of heaven.

Keywords: Dante, astronomy, purgatory, Southern Cross.

“... e quindi uscimmo a riveder le stelle", escribe Dante concluyendo la cántica del Inferno.
\end{abstract}

"Universidad Santo Tomás. Santiago, Chile. Correo electrónico: joblar@gmail.com Artículo recibido el 9 de abril de 2013. Aceptado el 4 de septiembre de 2013. 
Y son justamente las estrellas las que ve en el cielo sobre la playa del Antipurgatorio, que empieza a iluminarse con la luz del alba ventura.

"Dolce color d'oriental zaffiro, che s'accoglieva nel sereno aspetto

dal mezzo, puro infino al primo giro,

agli occhi miei ricominciò diletto,

tosto ch'i' usci' fuor de l'aura morta

che m'avea contristati gli occhi e 'l petto". (Pg I, 13-18).

[Dulce color de oriental zafiro, que era acogido por el medio en el sereno aspecto, puro hasta el primer el primer giro, recomenzó dilecto a mis ojos, tan pronto como yo salí fuera del aire muerto que me había entristecido los ojos y el pecho].

Es importante notar la lección dal mezzo porque -como observa Antonio Lanza en su edición crítica- del mezzo "no está en ningún códice de la antigua vulgata; por lo tanto hay que acoger la lección dal mezzo, entendiendo: 'un dulce color azulino, que estaba contenido (s'accoglieva) por la atmósfera (dal mezzo) en su serena apariencia, atmósfera pura hasta el horizonte'. Para mezzo en el significado de 'atmósfera' vd. Barbi, Problemi, I, pp. 216-7, pero que explica como la mayoría: 'en el sereno aspecto de la 'atmósfera'"'.

Precisamente acerca del mezzo [medio] -como término científico referido al fluido en medio al cual se encuentran y actúan los cuerpos (esto es, el aire)Dante había escrito en el Convivio:

"Visto este modo de la vista, se puede ver fácilmente que, ocurra que la estrella sea siempre de un modo luciente y clara, y no reciba mutación alguna sino de movimiento local, así como en aquel Del Cielo y del Mundo está probado, por más razones puede parecer no clara y no luciente. Porque puede parecer así por el medio que continuamente se transmuta. Se transmuta este medio de mucha luz en poca luz, así como a la presencia del sol y a su ausencia; y a la presencia el medio, que es diáfano, está tan lleno de luz que vence a la estrella, y por eso parece más luciente. Se transmuta también este medio de sutil a grueso, de seco a húmedo, por los vapores de la tierra que suben continuamente: el

${ }^{1}$ Alighieri, Dante. La Commedia, testo critico secondo i più antichi codici fiorentini a cura di Antonio Lanza. Anzio: De Rubeis, 1996, ad locum. Todas mis citas corresponden a este texto. Las traducciones al castellano me pertenecen. 
cual medio, así transmutado, transmuta la imagen de la estrella que viene por éste, por el grosor en obscuridad, y por lo húmedo y por lo seco en color"2.

El primo giro alude al primero entre los círculos de la esfera, el horizonte, que sirve a la determinación de todos los otros. Algunos, en cambio, han pensado en el cielo de la luna (Lana, Buti, Venturi) ${ }^{3}$, a la esfera del fuego (Benvenuto) ${ }^{4}$ o al primer móvil (Lombardi). En el caso de horizonte, se trata del horizonte visivo o sensible, esto es de la línea circular en la que el cielo parece confundirse con la tierra y que delimita la parte de suelo o de mar que podemos ver desde nuestro punto de observación. Esto se presenta como la base de una cúpula de aire azul. Y así lo confirma Pietro Alighieri en su Comentarium: "a medio usque ad primum gyro, id est horizontem" ${ }^{5}$.

En el cielo hay un signo astronómico clarísimo:

\section{Lo bel pianeto che d'amar conforta faceva tutto rider l'orïente, velando i Pesci ch'erano in sua scorta. (Pg I, 19-21).}

[El bello planeta que conforta a amar hacía reír todo el oriente, velando a los peces que estaban en su escolta].

Venus brilla en el Oriente en conjunción con la constelación de Piscis. Aceptando el significado moral de los versos (el ardor virtuoso que mueve a ese planeta "por el cual las almas de acá abajo se encienden de amor, según su disposición") hay también una referencia horaria. Como Piscis precede a Aries, en el cual -según Dante- se encontraba en ese momento el sol, quiere decir que

\footnotetext{
${ }^{2}$ Veduto questo modo della vista, vedere si può leggiermente che, avegna che la stella sempre sia d'un modo lucente e chiara, e non riceva mutazione alcuna se non di movimento locale, sì come in quello Di Cielo e Mondo è provato, per più cagioni puote parere non chiara e non lucente. Però puote parere cosi per lo mezzo che continuamente si transmuta. Transmutasi questo mezzo di molta luce in poca luce, sì come alla presenza del sole e alla sua assenza; e alla presenza lo mezzo, che è diafano, è tanto pieno di lume che è vincente della stella, e però pare più lucente. Transmutasi anche questo mezzo di sottile in grosso, di secco in umido, per li vapori della terra che continuamente salgono: lo quale mezzo, cosi transmutato, transmuta la imagine della stella che viene per esso, per la grossezza in oscuritade, e per l'umido e per lo secco in colore (Cv III ix 11-12).

${ }^{3}$ Lana, Iacopo della. Comedia di Dante degli Alighieri col commento di Iacopo della Lana bolognese, publicado por Luciano Scarabelli. Bologna: Tipografia Regia, 1866-67; Buti, Francesco da. Commento di Francesco da Buti sopra la Divina Commedia di Dante Alighieri, publicado por Crescentino Giannini. Pisa: Nistri, 1858-62; Venturi, Pompeo. La Divina Commedia di Dante Alighieri...col comento del P. Pompeo Venturi.Lucca: Cappurri, 1732.

${ }^{4}$ Benvenuto da Imola. Benvenuti de Rambaldis de Imola Comentum super Dantis Aldigherii Comoediam, publicado por James Philip Lacaita. Firenze: Barbèra, 1887.

5 "Desde el medio hasta el primer giro, éste es el horizonte". Alighieri, Pietro. Petri Allegherii super Dantis ipsius genitoris Comoedian commentarium, publicado por Vincenzo Nannucci. Firenze: Piatti, 1845.

${ }^{6}$ Per lo quale le anime di qua giuso s’accendono ad amore, secondo la loro disposizione (Cv II v 14).
} 
faltan cerca de dos horas para que surja el sol en el meridiano del Purgatorio. Está cercana el alba del cuarto día del viaje y -desde cuando han comenzado a salir del centro de la Tierra - han pasado poco más de veinte horas. En efecto, cuando habían pasado más allá del centro de la Tierra, Virgilio había dicho:

"Lèvati sù», disse 'l maestro, "in piede!

la via è lunga e 'l cammino è malvagio,

e già il sole a mezza terza riede». (Inf XXXIV, 94-96).

[«iPonte de pie!», dijo el maestro, «la vía es larga y el camino es malvado, y ya el sol vuelve a la media tercia»].

Esto es, después de hecho sentarse un momento a Dante, Virgilio le advierte que el camino por hacer es difícil y que son las siete y media de la mañana (la media tercia es la hora media entre la prima y la tercia hora canónica). Por otro lado, mientras miraban a Lucifer, el guía había advertido:

Ma la notte risurge, e oramai

è da partir, ché tutto avén veduto». (If XXXIV, 68-69).

[Pero la noche resurge, y ya hay que partir, que todo lo hemos visto].

En el hemisferio de Jerusalén -del cual partiero- anochece y, por lo tanto, en las antípodas -donde se encuentra la montaña del Purgatorio- es el comienzo del día. En ese momento, han transcurrido 24 horas desde el inicio del viaje, porque éste había empezado cuando:

Lo giorno se n'andava, e l'aere bruno

toglieva li animai che sono in terra

da le fatiche loro; ...(Inf II, 1-3).

[El día se marchaba, y el aire obscurecido quitaba sus fatigas a los seres animados que están en la tierra;]. 
Estamos, por lo tanto, en el atardecer del día después. En el momento que los dos poetas habían abandonado el puente de la novena bolgia, era una hora después del mediodía. Ocuparon algunas horas para visitar la décima bolgia y llegar al Cocito; después otras más para recorrer el radio completo del círculo nono.

La precedente indicación horaria había sido entregada por Virgilio cuando, precisamente, había llegado a la última bolgia del círculo octavo:

E già la luna è sotto i nostri piedi:

lo tempo è poco omai che nn'è concesso,

e altro è da veder che tu non vedi». (If XXIX, 10-12).

[Y ya la luna está bajo nuestros pies: ya es poco el tiempo que se nos ha concedido y otras cosas que tú no ves hay que ver»].

La luna está ahora en el nadir, en las antípodas de Jerusalén. Esto quiere decir que, estando en el meridiano del Purgatorio, es cerca de la una después de mediodía. De las palabras de Virgilio, resulta claro que el tiempo se acorta y -si quieren visitar el Infierno en 24 horas- ya han transcurrido 18 de ellas y quedan menos de seis.

Dejando la bolgia de los magos y adivinos (4a del círculo octavo), ya había advertido:

Ma vienne omai, ché già tiene 'l confine

d'amendue li emisperi e tocca l'onda

sotto Sobilia "Cain e le spine";

e già iernotte fu la luna tonda:

ben te n' dè ricordar, ché non ti nocque

alcuna volta per la selva fonda». (If XX, 124-129).

[Pero ven ahora, porque ya "Caín y las espinas" llega al confín de ambos los hemisferios bajo Sevilla; y ya ayer en la noche fue redonda la luna: bien te debes recordar de ello, que alguna vez no te dañó por la honda selva»]. 
Aquí también la luna era un punto de referencia, sobre la cual-siguiendo una imagen popular- se vería a Caín con un manojo de espinas (los signos obscuros... I que allá abajo en tierra / hacen a otros fabular de Cain, ${ }^{7}$ ). En ese momento, el satélite tocaba el mar bajo Sevilla (Sobilia) y se encontraba en el confín entre los dos hemisferios, esto es se ponía. Como la noche precedente había sido plenilunio (tonda) se podía calcular que eran las seis de la mañana. Non ti nocque quiere decir que la luz de la luna llena sirvió a Dante más de una vez mientras estaba extraviado en la selva obscura.

Es todo absolutamente coherente y es importante evidenciarlo porque Dante no deja nada a la improvisación. Los dos poetas entraron el séptimo círculo cuando eran alrededor de las tres horas antemeridianas del tercer día:

Ma seguim oramai, ché 'l gir mi piace;

ché ' Pesci guizzan su per l'orizonta,

e 'l Carro tutto sovra '1 Coro giace,

e 'l balzo via là oltre si dismonta». (If XI, 112-115).

[Pero sigamos ya, que el ir me place; porque los Peces se escurren arriba por el horizonte, y el Carro yace todo sobre el Coro, y la alta ribera mucho más allá empieza a descender].

A Virgilio place ir porque hay poco tiempo. Y él es el que indica la posición de la constelación de Piscis, que verán después en el cielo del Antipurgatorio. Mientras la Osa Mayor ( l Carro) está en dirección del viento maestral ('l Coro) que sopla desde Noroeste, las figuras zodiacales se mueven animalescamente sobre el horizonte tres horas antes del alba, precediendo a Aries que surge junto al sol. Sobre este último signo hay que recordar los famosos versos que aluden a la creación del mundo en el equinoccio de primavera:

Temp'era dal principio del mattino, che 'l sol montava sù con quelle stelle cheran co.lui quando l'amor divino mosse di prima quelle cose belle: (If I, 37-40).

7 li segni bui.../ che laggiuso in terra / fan di Cain favoleggiare altrui, (Pd II, 49-51). 
[Tiempo era ya del principio de la mañana, que el sol montaba arriba con esas estrellas que estaban con él cuando el amor divino movió primero esas cosas bellas:]

Visitaron rápidamente el círculo de los violentos y las primeras cuatro bolgias del Círculo VII. Como apenas he recordado, se encontraron en el borde que divide la cuarta de la quinta bolgia cuando la luna se ponía en el horizonte. Esto quiere decir que el sol se había alzado desde hacía una hora. En cambio, cuando se disponían a pasar sobre el puente de la quinta bolgia, eran cerca de las seis antemeridianas. Lo confirma Malacoda recordando la hora de la muerte del Cristo:

Ier, più oltre cinque ore che quest'otta,

milledugento con sessantasei

anni compié che qui la via fu rotta. (If XXI, 112-114).

[Ayer, cinco horas más allá de esta hora, mil doscientos con sesenta y seis años se cumplieron que aquí la vía se rompió].

El cálculo es simple. Si se considera que Jesús murió a los 34 años ("ab incarnatione"), la suma de 1266 + 34 da 1300, que es el Annus Domini en el que se desarrolla el viaje de Dante ${ }^{8}$. Si faltan cinco horas, es necesario hacer el cálculo con la información en la que creía Dante y esto es:

"Y me mueve esta razón: que óptimamente naturado fue nuestro salvador Cristo, el cual quiso morir en el trigésimo cuarto ańo de su edad; porque no era conveniente para la divinidad hallarse en situación de decrecer; ni hay que creer que él no quisiese morar en esta vida nuestra al sumo, después que había estado de la puericia. Y esto manifiesta la hora del día de su muerte, esto es de Cristo, que quiso aquella semejar con la vida suya: por lo que dice Lucas que era casi la hora sexta cuando murió, que es decir el colmo del día. Por lo que se puede comprender por ese "casi" que en el trigésimo quinto año de Cristo estaba en el colmo de su edad" 9 .

\footnotetext{
${ }_{8}^{8}$ No hay dudas sobre el año del viaje. Para toda la cuestión, cfr. mi síntesis en Blanco Jiménez, José. "Bene ascolta chi la nota" (If. XV, 99). Intertextualidad clásica en la Commedia de Dante. Santiago de Chile: Ediciones Video Carta, 2007.

9 E muovemi questa ragione: che ottimamente naturato fue lo nostro salvatore Cristo, lo quale volle morire nel trentaquattresimo anno della sua etade; ché non era convenevole la divinitade stare in cosa [in] discrescere; né da credere è ch'elli non volesse dimorare in questa nostra vita al sommo, poi che stato c'era nel basso stato della puerizia. E ciò manifesta l'ora del giorno della sua morte, cioè di Cristo, che volle quella consimigliare colla vita sua: onde dice Luca che era quasi ora sesta quando morìo, che è a dire lo colmo del die. Onde si può comprendere per quello "quasi" che al trentacinquesimo anno di Cristo era lo colmo della sua etade (Cv IV XXIII, 10-11).
} 
Esto quiere decir que ha pasado una hora desde cuando los dos poetas habían llegado al puente de la 5a bolgia (If XX, 124-126) y, por lo tanto, son las siete de la mańana.

Y hay otras dos menciones horarias.

Cuando estaban llegando a la ciénaga Estigia, Virgilio había dicho:

Or discendiamo omai a maggior pièta;

già ogni stella cade che saliva

quand'io mi mossi: 'l troppo star si vieta. (If VII, 97-99).

[Ahora descendamos ya a mayor piedad; ya cae cada estrella que subía cuando yo me moví: se prohíbe el estar demasiado].

Para que todas las estrellas que subían en el hemisferio oriental (con respecto al meridiano de Jerusalén) hayan pasado al hemisferio occidental (caen, bajan) deben haber transcurrido doce horas. El viaje de Dante empezó después del ocaso (cfr. cit. If II, 1-3) y, por lo tanto, la referencia cronológica tiene que ver con el momento en que Virgilio se movió del Limbo. Haciendo un cálculo entre el ocaso y la hora de If XI, 113-114, se puede acercar a la medianoche. En todo caso - lo señalo desde ya- es un dato que ayuda a confirmar las dos fases de composición de la Commedia.

Más simple es la información que Dante entrega a Brunetto Latini, asegurando que había dejado la selva obscura:

Pur ier mattina le volsi le spalle: (If XV, 52),

[Sólo ayer en la mañana le volví las espaldas:]

Cuando se detuvieron a hablar con Malacoda, faltaban cinco horas para el mediodía (If XXI, 115). Después tuvieron varios encuentros en las diversas bolgias (Ciampolo di Navarra, Catalano dei Catalani, Vanni Fucci, Ulisse, Guido da Montefeltro, Maometto, Pier da Medicina, Mosca de' Lamberti, Bertram dal Bornio) y habían pasado otras seis.

Todo claro, por lo tanto, en lo que a la hora se refiere. No así en cuanto se refiere al día. 
Si observamos el denominado "reloj" de Edward Moore ${ }^{10}$, hasta ese momento, el viaje se ha desarrollado en los siguientes días, lugares (episodio) y horas:

- jueves 7 de abril: selva obscura (noche entera, If I, 21)

- viernes 8 de abril: las tres fieras (día entero, If I, 37ss.); Círculo I (caer de la noche, If II, 1); Círculo IV (medianoche, If VII, 98).

- sábado 9 de abril: Círculo VI (alrededor de las 3.00 AM, If XI 113); Círculo VIII bolgia $3^{\mathrm{a}}$ (inmediatamente después del aparecer del sol, alrededor de las 6.00-6.30 AM, If XX, 125); Círculo VIII 5a bolgia (alrededor de las 7.00 AM, If XXI, 112); Círculo VIII 9a bolgia (alrededor de la 1.00 PM, If XXIX, 10); Giudecca (7.30 PM y -apenas pasado el centro de la Tierra- 7.30 AM, If XXXIV, 96 y $101-105)$.

- domingo 10 de abril (Día de Pascua): antipurgatorio (entre las 4.00 y las 5.00 AM, Pg I, 19-21; poco antes de que aparezca el sol, Pg I, 107-115).

Por su parte, Camilli, propone el inicio del viaje el 25 de marzo ${ }^{11}$ y la llegada a la playa del Purgatorio sería en el alba del día 27. Yo, después de haber meditado y calculado largamente acerca de la economía cronológica del poema, me inclino en cambio por la solución de Mirco Manuguerra, según el cual el viaje comienza el 4 de abril y, por lo tanto, la llegada al Antipurgatorio se debe fechar el 7 de abril. Él sostiene que la luna tonda no quiere decir necesariamente plenilunio y que Malacoda (embustero y padre de la mentira, If XXIII, 144 ${ }^{12}$ ) miente en lo que se refiere al día, así como lo ha hecho con los efectos del terremoto. Virgilio (y Dante) no se han dado cuenta porque están demasiado preocupados acerca de si habrían de lograr la prosecución del viaje $\mathrm{e}^{13}$.

Pero debo pasar al segundo argumento que me interesa de esta visión del cielo del Antipurgatorio. Junto a las precisas indicaciones astronómicas ya comentadas, Dante agrega:

\footnotetext{
${ }^{10}$ Moore, Edward. Gli accenni al tempo nella Divina Commedia e loro relazione con la presunta data e durata della visione, traducción italiana de Cino Chiarini. Roma: Salerno, 2007. El texto está tomado de Studies in Dante, Third Series: Miscellaneous Essays, Oxford: Clarendon, 1968 [1903], pp.144ss. Es un dantólogo inglés (Cardiff 1835 - Oxford 1916), fue el fundador de la Sociedad Dantesca de Oxford y canónigo de Canterbury desde 1903.

${ }^{11}$ Camilli, Amerindo. "La cronologia del viaggio dantesco", Studi Danteschi XXIX (1950): 61-84. es sobre todo un fonetista (Servigliano, 1879-1960). Es sobre todo un fonetista (Servigliano, 1879-1960).

${ }^{12}$ bugiardo e padre di menzogna (If XXIII, 144).

13 Manuguerra, Mirco. "La fisica di Dante e l'enigma astronomico della datazione del viaggio nella Divina Commedia". Atti del XVII Congresso Nazionale di Storia della Fisica e dell'Astronomia, C.N.R. - Consiglio Nazionale delle Ricerche, Commissione di Studio per la Storia della Fisica e dell'Astronomia, Como, Centro Volta - Villa Olmo, 23-24 maggio 1997. Ver después IDEM, "Una soluzione teologico-astronomica coerente per l'enigma della datazione del Viaggio nella Commedia”, L'Alighieri XLIV, n. 21. Es el presidente del Centro Lunigianese di Studi Danteschi, con sede en Mulazzo (Massa Carrara, Italia).
} 


\section{I' mi volsi a man destra, e puosi mente}

a l'altro polo: e vidi quattro stelle

non viste mai, fuor ch'a la prima gente. (Pg, I, 22-24).

[Yo me di vuelta a mano derecha, y puse mente al otro polo: y vi cuatro estrellas no vistas nunca, fuera que por la primera gente].

En el Purgatorio, Dante irá siempre hacia la derecha, mientras en el Infierno iba a la izquierda. El otro polo es el antártico, que desde el Purgatorio se ve más o menos 30 grados sobre el horizonte. Del mismo modo, se puede observar el polo ártico desde Jerusalén (que se encuentra a 310 46’ de latitud Norte y 350 14 ' de longitud Este). Aclarado el tema del paralelo, resulta interesante hacer notar que, en la geografía de Dante, el meridiano de Jerusalén equivale al que ahora es el de Greenwich. Esto quiere decir que la montaña de la expiación está ubicada en el meridiano de cambio de fecha.

Vistas las referencias a constelaciones que existen verdaderamente (el poeta recordará nuevamente la Osa Mayor en el v. $\left.30^{14}\right)$, muchos han querido que esas cuatro estrellas hayan estado verdaderamente en el cielo meridional. Por ello, alguno incluso ha pensado en la Cruz del Sur.

Aunque el texto dantesco es muy claro a este respecto, no está demás escribir algunas palabras sobre este asunto.

Astronómicamente, la denominada Cruz del Sur figura, con el nombre de Pies del Centauro, en el catálogo de Tolomeo, que vivía en Alejandría de Egipto. En efecto, estas estrellas son visibles desde el África del Norte y los Romanos las llamaban el Trono de César. Se veían sobre el horizonte en Jerusalén cuando murió Jesús Cristo, pero después descendió poco a poco por la precesión de los equinoccios. En Alejandría de Egipto, se veían en el 1300 y podían verlas los peregrinos que iban a Tierra Santa antes de las Cruzadas, que se desarrollaron

\footnotetext{
${ }^{14}$ Con respecto al Carro, me parece interesante traducir parte de lo que Giovanni Buti y Renzo Bertagni en Enciclopedia dantesca, Roma: Treccani, 1970, I, 851: "Si en las latitudes más allá de los $40^{\circ}$ la Osa Mayor no se pone nunca, ¿cómo se comporta a 32 de latitud boreal (Jerusalén) o austral (Purgatorio)? En Jerusalén está alta sobre el horizonte cerca de 18 horas y se pone por alrededor de 6 horas, y una sola de sus estrellas (la $\alpha$ ) se ve siempre; de converso, en las antípodas, o sea en el Purgatorio, está alta sobre el horizonte alrededor de 6 horas y la $\alpha$ no se ve nunca. Estas dos situaciones opuestas, no expresas, quedan implícitas en If XI 114 y en $\mathrm{Pg}$ 129-30, que resultan dos pasos análogos: en efecto, en el primero se habla del próximo ocaso del C (arro) por Jerusalén y en el segundo de desaparición ya ocurrida para el Purgatorio. D(ante) habla del C(arro), en el primer caso (If XI), para describir el alba, con los Peces que surgen sobre el horizonte de Jerusalén mientras el C(arro) se dirige al ocaso (y el Carro todo sobre el Coro yace, donde el Coro es viento de noroeste), porque el C(arro), diferiendo en ascensión recta de los Peces de alrededor de $12 \mathrm{~h}$ en media y teniendo una declinación media de alrededor de $55^{\circ}$, en el punto del ocaso tiene un azimut de alrededor de $35^{\circ}$, lo que lo lleva a estar en el cielo de noroeste. En el segundo caso (Pg I) D (ante) cumple un giro de horizonte desde el Purgatorio: se vuelve al sur, oeste y norte allá donde el Carro ya había desaparecido, por lo que la referencia al C(arro) es asaz natural".
} 
entre el 1095 y 1271 . No sabemos si San Francisco fue verdaderamente a Damietta (Egipto), que se encuentra cerca de la misma latitud de Alejandría pero, si la hubiese visto, este hecho seguramente habría sido transmitido por las tradiciones franciscanas.

Fue el toscano Andrea Corsali, de Émpoli, quien quiso ver en esa constelación el símbolo de la Cristiandad. Después de haber hecho -por cuenta de los Medici- un largo viaje de circunnavegación de África llegando hasta la India, escribió en 1516 la siguiente relación: "Después que partimos de Lisboa navegamos siempre con viento próspero, no saliendo de siroco y lebeche y pasando la línea equinoccial fuimos a la altura de treinta y siete grados en el otro hemisferio, a través del cabo de Buena Esperanza, clima ventoso, que en esos tiempos el sol se encontraba en los signos septentrionales, y encontramos la noche de 15 horas. Aquí vimos un admirable orden de estrellas, que en la parte del cielo opuesta a nuestra tramontana van girando infinitas. En que lugar esté el polo antártico, por la altura de los grados, tomamos el día con el sol y reencontramos la noche con el astrolabio, y evidentemente lo manifiestan dos nubecillas de tamaño razonable, que en torno a ella continuamente ahora bajándose ahora alzándose caminan con movimiento circular, con una estrella siempre en el medio, la cual con ellas se vuelva alrededor de once grados lejos del polo. Sobre éstas aparece una cruz maravillosa en el medio de cinco estrellas, que la circundan (como la Tramontana al Carro) con otras estrellas, que con ellas van girando en torno al polo lejos alrededor de treinta grados: y hace su curso en 24 horas, y es de tanta belleza que no me parece poderla comparar a signo celeste alguno, como en la forma que aparece aquí más abajo" ${ }^{15}$.

Y agrega un dibujo en el cual -además de las denominadas "nubes de Magallanes"- se advierten las estrellas que hoy aún sobresalen en las banderas de Australia, Nueva Zelanda, Papua-Nuova Guinea, Samoa y de Brasil. En todo caso, queda claro que las estrellas son cinco y no cuatro.

\footnotetext{
15 Dapoi che partimmo da Lisbona navigammo sempre con prospero vento, non uscendo da scilocco e libeccio, e passando la linea equinoziale fummo in altura di trentasette gradi nell'altro emispero, a traverso di capo di Buona Speranza, clima ventoso e freddo, ch'a quei tempi il sole si trovava ne' segni settentrionali, e trovammo la notte di 14 ore. Qui vedemmo un mirabil ordine di stelle, che nella parte del cielo opposita alla nostra tramontana infinite vanno girando. In che luogo sia il polo antartico, per l'altura de' gradi, pigliammo il giorno col sole e ricontrammo la notte con l'astrolabio, ed evidentemente lo manifestano due nugolette di ragionevol grandezza, chintorno ad essa continuamente ora abbassandosi e ora alzandosi in moto circulare camminano, con una stella sempre nel mezzo, la qual con esse si volge lontana dal polo circa undici gradi. Sopra di queste apparisce una croce maravigliosa nel mezzo di cinque stelle, che la circondano (com'il Carro la Tramontana) con altre stelle, che con esse vanno intorno al polo girandole lontano circa trenta gradi: e fa suo corso in 24 ore, ed è di tanta bellezza che non mi pare ad alcuno segno celeste doverla comparare, come nella forma qui di sotto appare (El texto está traducido de la edición de la carta escrita por Andrea Corsali, el 6 de enero de 1515 - según el uso florentino - y publicada por Giovan Battista Ramusio en sus Navigationi et viaggi. Venezia: Lucantonio Giunti, 1550, vol. I, f. $192 \mathrm{v}^{\circ}$.
} 
También Antonio Pigafetta quiso, en 1524, escribir en su Relación del primer viaje en torno al mundo: "El polo Antártico no es tan estrellado como el Ártico. Se ve muchas estrellas pequeñas, congregadas juntas, a manera de dos nubes poco separadas la una de la otra y un poco ofuscadas, en medio de las cuales hay dos estrellas muy grandes, ni muy relucientes y que poco se mueven. Nuestra calamita, desviando uno siempre, tiraba a su polo Ártico; nada menos no tenía tanta fuerza como de la banda suya. Y por eso, cuando estábamos en este golfo, el capitán general preguntó a todos los pilotos, yendo siempre a vela, por cuál camino navegando marcábamos en las cartas. Respondieron todos: Por la vía marcada por él: les respondió que marcaban falso, así como era, y que convenía ayudar a la aguja de navegación, porque no recibía tanta fuerza de su parte. Cuando estábamos en este golfo vimos una cruz de cinco estrellas lucidísimas, derecho al poniente y son justísimas la una con la otra" ${ }^{16}$ Nuevamente la referencia corresponde a cinco estrellas y no a cuatro.

En cuanto a la mención que hace Amerigo Vespucci -en su carta del 18 de julio de 1500 dirigida a Lorenzo di Pierfrancesco de’ Medici- la influencia de Dante está declarada de manera expresa, dado que transcribe los versos de Pg I, 21-27 y comenta: "Que según yo, me parece que el Poeta en estos versos quiera describir, por las cuatro estrellas, el polo del otro Firmamento, y no desconfío hasta aquí que lo que dice no sea la verdad, porque yo noté cuatro estrellas figuradas como una almendra, que tenía poco movimiento; y si Dios me da vida y salud, espero pronto volver a es hemisferio y no volver sin anotar el polo. ${ }^{17}$

¿Especulaciones? Muchísimas y cito algunas para demostrar su inconsistencia.

Ciertamente, en el Almagesto de Tolomeo (s. II d.C.; que sin embargo es copia del perdido catálogo estelar de Hiparco de Nicea, que es del s. III a.C.) ya estaban indicadas las estrellas del Centauro, pero no nombra cruz alguna. Ese

\footnotetext{
${ }^{16}$ Il polo Antartico non è cosi stellato come lo Artico. Se vede molte stelle piccole, congregate insieme, che fanno in guisa de due nebule poco separate l'una dall'altra e uno poco offusche, in mezzo delle quale stanno due stelle molto grandi, nè molto relucenti e poco se moveno. La calamita nostra, zavariando uno sempre, tirava al suo polo Artico; niente de meno non aveva tanta forza come da la banda sua. E però, quando èramo in questo golfo, il capitano generale domandò a tutti li piloti, andando sempre a la vela, per qual cammino navigando pontasseno su le carte. Risposero tutti: Per la sua via puntualmente data: li rispose che pontavano falso, cosi come era, e che conveniva aiutare la guglia del navigare, perchè non riceveva tanta forza dalla parte sua. Quando èramo in questo golfo vedessimo una croce de cinque stelle lucidissime, dritto al ponente e sono giustissime una con l'altra. (El texto está traducido de Primo viaggio intorno a globo terracqueo ossia Ragguaglio della Navigazione alle Inde Orientali per la via d'occidente fatta dal cavalier Antonio Pigafetta... tratta da un Codice MS della Biblioteca Ambrosiana di Milano e corredata di note da Carlo Amoretti. Milano: Stamperia di Giuseppe Galeazzi, 1800, p. 47.

${ }^{17}$ Che secondo me, mi pare che il Poeta in questi versi voglia descrivere, per le quattro stelle, il polo dell'altro Firmamento, $e$ non mi diffido fino a qui che quello che dice, non sia la verità, perchè io notai quattro stelle figurate come una mandorla, che tenevano poco movimento; e se Dio mi dà vita e salute, spero presto tornare in quello emisperio e non tornar senza notare il polo (El texto está traducido de la edición de Vespucci, Amerigo, Lettere di viaggio, publicada por Formisano, Luciano. Milano: Mondadori, pp. 3-7).
} 
nombre es una invención del astrónomo francés Augustin Royer que, en su atlas celeste de 1679, la separó del Centauro.

Seguramente, los árabes habían dibujado mapas del cielo austral, pero no hay pruebas de que Dante las conociera. Entonces, hay quien sostiene que Brunetto Latini habría podido ver una carta astral en España (por lo menos, existe un mapa de 1154 que reporta gran parte del África y del Océano Índico) y después ser Brunetto habría hablado de ella con su discípulo Dante.

Las primeras representaciones cartográficas conocidas de la denominada Cruz del Sur son las de Petrus Plancius (1598) y de Jodocus Hondius (1600). Por otro lado, resulta visible sólo desde la extremidad meridional de la península del Sinaí, o si no desde las Islas Canarias (25 latitud Norte). Y, por lo que sabemos de sus escritos, Dante no llegó nunca más al sur de Roma.

¿Podría haber alguna fuente literaria? En la Odisea-compuesta en el siglo VIII a.C. y que Dante no conoció- Calipso da algunas indicaciones astronómicas a Ulises (V, 269-281), pero de la cruz no hay mención alguna.

El mismo Dante hace narrar a Ulises el que habría sido su último viaje y éste declara:

«Tutte le stella già de lıaltro polo vedea la Notte, e I nostro tanto basso

ch'e' non surgëa fuor del marin suolo». (If XXVI, 127-129).

[ YYa todas las estrellas del otro polo veía la Noche, y el nuestro tan bajo que él no surgía fuera del suelo marino»].

Si "nuestro" polo (esto es, el polo ártico) era tan bajo que no surgía por sobre el horizonte marino, quiere decir que había pasado el ecuador y se encontraba por lo menos a $40^{\circ}$ de latitud Sur. Por ello veía todas las estrellas del hemisferio austral, pero no las circumpolares septentrionales.

Un solo detalle que se le ha escapado a muchos: Ulises habría llegado casi diezgrados más al Sur de donde Dante coloca la montaña del Purgatorio. Además, se demoró cinco meses en llegar (La luz se habia encendido cinco veces y otras tantas cancelada bajo de la luna, después que habiamos entrado en el alto paso ${ }^{18}$ ) y ésta es otra eventual incoherencia. Sin embargo, el Carro de la Osa Mayor es la constelación más importante del cielo europeo y su desaparición habría generado un cierto ${ }_{18}$ Cinque volte racceso e tante casso / lo lum era di sotto da la luna / poi che 'ntrati eravam ne l'alto passo (If XXVI, 130-132). 
desaliento en algunos viajeros (como los soldados de Alejandro Magno lejos de sus hogares $^{19}$ o Marco Polo en la isla de Sumatra ${ }^{20}$ ) que veían desaparecer un punto de orientación. Entonces, puede decir que es probabilísimo que Ulises haya dado vuelta en banda por tanto tiempo precisamente porque no tenía la estrella polar como referencia. Además, si bien Dante no conoció la Odisea, seguramente sabía que había sido un viaje arduo, porque había durado siete años, aunque el punto de partida (Troya) y el de llegada (Itaca) no estuvieran tan lejos en línea de aire.

Un observador colocado en el ecuador (la latitud cero) puede ver, teóricamente, todas las estrellas del cielo, porque ambos polos son visibles. Y en las regiones ecuatoriales habitaban algunos pueblos. Dante-efectuando cálculos de tipo astronómico- recuerda a los Garamantes, que estaban en la Phazania romana (actual Fezzàn, en el Sur de Libia, entre el ecuador y el grado 16º y que controlaban las caravanas a través del desierto del Sahara. Se refiere a ellos dos veces en el Convivio ${ }^{21}$

\footnotetext{
19 Iam nec flumina posse adiri nec sidera pristinum servare fulgores (Ya no se podían pasar los ríos ni los astros mantenían su primitivo fulgor; Curtius Rufis, Quintus. Historiarum Alexandri Magni Macedonis libri qui supersunt, IV Cap. 10, 3; publicado por Vogel, Theodor. Leipzig: Teubner, 1903-1906. Reimpreso en 2002.

${ }_{20}$ ed io Marco Polo vi dimorai cinque mesi per lo mal tempo che mi vi teneva; e ancora la tramontana non si vedeva ne le stelle del maestro (y yo Marco Polo moré allí cinco meses por el mal tiempo que allí me retenía; y aún la tramontana se veía en las estrellas del maestro; otro códice lee un anno, o sea "un ańo"). Sigo y traduzco el texto de Il milione di Marco Polo: testo di lingua del secolo decimoterzo ora per la prima volta pubblicato ed illustrato dal conte Gio. Battista Baldelli-Boni, Firenze: da' torchi di G. Pagani, 1826, p. 162. El curador agrega en nota: Pare che voglia significare che non vedeva la stella polare. Quanto a ciò che esso rozzamente appella stella del Maestro, a parere del Chiaro Padre Inghirami sembra che voglia significare il Carro di Boote, che nei luoghi ove si nasconde sotto l'orizzonte, tramonta verso la parte di Maestro. Esso non vedendo detta costellazione, dimostra che si trovava nel Reame di Samarcha nel tempo in cui questa costellazione percorre nella notte la parte inferiore del suo arco diurno, e perciò in quei climi dovea trovarsi nella notte sotto 1'orizzonte (Parece que quiera significar que no veía la estrella polar. En cuanto a lo que él toscamente llama estrella del Maestro, según el parecer del Claro Padre Inghirami parece que quiera significar el Carro de Boote, que en los lugares donde se esconde bajo el horizonte, se pone hacia la parte del Maestro. Él no viendo dicha constelación, demuestra que se encontraba en el Reino de Samarcha [Sumatra] en el tiempo en el que esta constelación recorre en la noche la parte inferior de su arco diurno, y por ello en estos climas debía encontrarse en la noche bajo el horizonte).

21 vez Imaginisi anche uno cerchio in su questa palla, che sia in ciascuna parte sua tanto lungi da Maria quanto da Lucia. Credo che questo cerchio - secondo ch'io comprendo per le sentenze delli astrologi e per quella d'Alberto della Magna nel libro della Natura dei luoghi e [in quello] delle Propietadi delli elementi, e anco per la testimonianza di Lucano nel nono suo libro dividerebbe questa terra discoperta dal mare Occeano, là nel mezzo die, quasi per tutta l'estremità del primo climate, dove sono intra l'altre genti li Garamanti, che stanno sempre quasi nudi; alli quali venne Catone col popolo di Roma, la segnoria di Cesare fuggendo (Imagínese también un círculo sobre esta esfera, que esté en cada parte suya tan lejos de María como de Lucía. Creo que este círculo - según lo que yo comprendo por la sentencias de los astrólogos y por aquella de Alberto de la Magna en el libro de la Naturaleza de los lugares y [en aquél] de las Propiedades de los elementos, y también por el testimonio de Lucano en el nono de su libro - dividiría esta tierra descubierta del mar Océano, alla en el mediodía, casi por toda la extremidad del primer clima, donde están entre las otras gentes los Garamantes, que están siempre casi desnudos; a los cuales vino Catón con el pueblo de Roma, huyendo de la señoría de César; Cv III v 12); Conviene anche che lo cerchio dove sono li Garamanti, come detto è, in su questa palla, veggia lo sole a punto sopra sé girare, non a modo di mola, ma di rota; la quale non può in alcuna parte vedere se non mezza, quando va sotto l'Ariete. E poi lo vede partire da sé e venire verso Maria novanta e uno die e poco più, e per altrettanti a sé tornare; e poi, quando è tornato, va sotto la Libra, e anche si parte e va ver Lucia novanta e uno die e poco più, e in altrettanti ritorna. (Conviene también que el círculo donde están los Garamantes, como se ha dicho, sobre esta esfera, vea el sol a punto girar sobre sí, no a modo de muela, sino de rueda; la cual no puede ver en parte alguna sino media, cuando va bajo Aries. E después lo ve partir de sí y venir hacia María noventa y un días o poco más, y por otros tantos volver a sí; y después, cuando ha vuelto, va bajo Libra, y también se parte y va hacia Lucía noventa y un días y poco más y otros tantos retorna; Cv III v 18). Dante usa los nombre de María y Lucía para indicar dos ciudades hipotéticas, puesta la una a las antípodas de la otra, en el polo antártico y en el polo ártico.
} 
y una vez en la Monarchia, donde los contrapone a los Escitas. ${ }^{22}$ Ellos pueden ver la Cruz del Sur, pero -como ya dije- se podía ver también a lo largo del valle del Nilo, a partir del paralelo $27^{\circ}$ de latitud Norte.

Además, Dante conocía el fenómeno de la precesión de los equinoccios. ${ }^{23}$

Sabía, por lo tanto, que -por razones astronómicas- las estrellas visibles en ciertas latitudes un tiempo pueden ya no serlo siglos después.

Giovanni Virginio Schiaparelli llegó a imaginar que la constelación había sido recordada en el Libro de Job, que es del siglo IV a.C. En efecto, en el texto de la Vulgata se puede leer: "Qui facit Arcturum et Oriona, et Hyadas et interiora austri" (Job 9, 9). Esto es: "Crea la Osa y el Orión, las Pléyades y los cuartos interiores del cielo austral” 24 . Pero, ¿qué quiere decir? Además, el hecho de que las cuatro estrellas se pudiesen ver desde la Palestina meridional siglos antes del nacimiento del Cristo no es "certificación" de absolutamente nada.

\footnotetext{
${ }_{22}$ Aliter quippe regulari oportet Scithas qui, extra septimum clima viventes et magnam dierum et noctium inequalitatem patientes, intolerabili quasi algore frigoris premuntur, et aliter Garamantes qui, sub equinoctiali habitantes et coequatam semper lucem diurnam noctis tenebris habentes, ob estus aeris nimietatem vestimentis operiri non possunt. (De otro modo, sin duda, es preciso que deban ser regulados los Escitas los cuales, viviendo fuera del séptimo clima y padeciendo la magna desigualdad de días y noches, están oprimidos por un frío glacial casi intolerable y, de otro modo, los Garamantes, los cuales, habitando bajo la zona equinoccial y teniendo siempre la luz del día equitativa a las tinieblas de la noche, no pueden ir vestidos debido al aire caliente en exceso; Mn I xiv 6). El séptimo clima - según Alberto Magno - está ubicado entre los grados 45 y 48 y corresponde a la zona más gélida del planeta. Toda la fuente se encuentra en De natura locorum, III, 38. Otra referencia podría encontrarse en este paso de la Questio de aqua et terra: Per lineam vero latitudinis, ut comuniter habemus ab eisdem, extenditur ab illis quorum cenith est circulus equinoctialis, usque ad illos quorum cenith est circulus descriptus a polo zodiaci circa polum mundi, qui quidem distat a polo mundi circiter xxiij gradus; et sic extensio latitudinis est quasi lxvij graduum et non ultra, ut patet intuenti. (Con respecto a la línea de latitud, según los susodichos generalmente consideran, se extiende desde aquellos [pueblos] cuyo cenit es el círculo equinoccial hasta aquéllos cuyo cenit está en el círculo descrito que el polo del zodíaco describe en torno al polo del mundo, el cual polo del zodíaco dista del polo del mundo cerca de 23 grados; y así la extensión de la latitud es casi de 67 grados y no más, como advierte quien mira; Q 55).

${ }^{23}$ Éste es argumento para otro artículo. Mientras tanto se puede cfr. Cv II iii 5; II v 17; II xiv 1, 11, 16; III vi 2-3; Vn II 2; y Pd XXVII, 142-148. De todos modos, según los cálculos que han sido efectuados, lac denominada "Cruz del Sur" culminó bajo el horizonte italiano entre el 700 a.C. y el 100 a. C. Los navegantes la habrían "redescubierto" cuando comenzaron a viajar por el hemisferio Sur. El único inconveniente es que hay que conocer el símbolo cristiano para identificarlo como tal y -antes de la crucifixión de Jesús- no es posible por razones obvias.

24 El astrónomo italiano analiza el versículo bíblico (cfr. Scritti sulla Storia dell'Astronomia Antica, I, pp.133-143; texto publicado en 1903 y ahora consultable en www.liberliber.it) y llega a la siguiente conclusión: Negli anni 750 avanti Cristo tutta questa regione passava al meridiano sull'ultimo orizzonte australe di Palestina, le stelle brillanti sopra accennate culminando ad altezze comprese fra $5^{\circ}$ e $16^{\circ}$. Tali stelle formavano e formano una costellazione grandiosa e più splendida di qualunque altra, Orione non escluso; la quale sulle carte odierne è divisa fra la Nave Argo, la Croce del Sud, ed il Centauro. Questa è la costellazione, che possiamo con tutta probabilità identificare cogli interiora Austri; non solo perchè soddisfa, ma anche perchè unica soddisfa a tutte le condizioni del caso. Nei tempi a cui qui si allude, i pastori e gli agricoltori della Palestina hanno potuto (ciò che ora i loro successori più non possono) contemplarla all'orizzonte estremo meridionale sotto aspetto di luce intensa quasi di aurora australe, cospersa di stelle brillanti, ed ammirare uno spettacolo, che oggi si può vedere soltanto da chi discenda verso l'Equatore fino al $20^{\circ}$ parallelo circa di latitudine Nord (En los años 750 antes de Cristo toda esta región pasaba al meridiano sobre el último horizonte austral de Palestina, las estrellas brillantes indicadas más arriba culminando a las alturas comprendidas entre el $5^{\circ}$ y el $16^{\circ}$. Tales estrellas formaban y forman una constelación grandiosa y más espléndida que cualquier otra, sin excluir Orión; la cual en los mapas actuales está dividida entre la Nave Argos, la Cruz del Sur, y el Centauro. Ésta es la constelación que, con toda probabilidad, podemos identificar con los interiora Austri; no sólo porque satisface, sino también porque es la única que satisface a todas las condiciones del caso. En los tiempos a los cuales aquí se alude, los pastores y los agricultores de la Palestina han podido (lo que ahora sus sucesores ya no pueden) contemplarla en el horizonte extremo meridional bajo el aspecto de luz intensa casi de aurora austral, esparcida de estrellas brillantes, y admirar un espectáculo, que hoy puede ser visto sólo por quien descienda hacia el Ecuador hasta aproximadamente el paralelo $20^{\circ}$ de latitud Norte; pp. 141-142).
} 
Por lo tanto, esas estrellas son una invención de Dante. ¿Pero por qué, para algunos, eso parece casi un pecado mortal? Para mí, la respuesta es simple: porque querríamos que todo lo que se lee en el poema sea verdadero y - además - porque Dante sabía de astronomía como lo demuestra en el Convivio y en la Questio de aqua et terra.

Dante necesitaba expresar una alegoría de las virtudes cardinales y de las virtudes teologales y su solución estuvo en inventar estrellas en la zona circumpolar invisible. ¿Pero -además del hecho de que el número expresa la plenitud- por qué inventó siete puntos luminosos?

Hay una respuesta que me parece la más adecuada.

El sistema tolemaico había sido introducido en Occidente por la traducción del Almagesto, ejecutada de la lengua árabe por Gerardo di Cremona en 1175. La Iglesia Católica Apostólica Romana, que había adherido a éste, veía en él una demostración de la simetría divina. Hay que pensar, por lo tanto, que si cerca del Polo Norte se encontraban las siete estrellas de la Osa Mayor (de donde viene, justamente, el vocablo septentrión), Dante podía pensar que existiesen otras siete en el Polo Sur. Sólo que están distribuidas en una constelación de cuatro y en otra de tres.

En efecto, más adelante, los viajeros verán otras tres estrellas que se encuentran en la parte opuesta con respecto al Polo Sur celeste:

\section{E'l duca mio: "Figliuol, che lassù guarde?».}

E io a lui: "A quelle tre facelle

di che 'l polo di qua tutto quanto arde».

Ond'elli a me: "Le quattro chiare stelle

che vedevi staman, son di là basse,

e queste son salite ov'eran quelle». (Pg VIII, 88-93).

[Y mi guía: «Hijo, ¿qué miras allá arriba?»Y yo a él: «Aquellas tres antorchas por las que el polo acá arde completamente». Por lo que él a mí: «Las cuatro luminosas estrellas que veías esta mañana, están allá bajas, y éstas han subido donde estaban aquéllas».] 
Esto es, Virgilio recuerda de manera explícita las que vieron en el alba. Se trata, claramente, de las virtudes teologales (fe, esperanza y caridad) en contraposición a las cardinales (prudencia, justicia, templanza, fortaleza). Así lo creyeron también los comentadores más antiguos: el Lana, el Ottimo, Benvenuto, Buti y el Anónimo Florentino ${ }^{25}$. Desde el punto de vista alegórico, el grupo de tres -que animan la vida contemplativa- aparecerían de noche, iluminando a las almas dedicadas a la meditación y a la plegaria. En cambio, el grupo de cuatro -que forman parte de la vida activa- iluminan a las almas junto con el surgir del día. Y un detalle más: en ningún momento Dante dice que forman una cruz y Corsali, en su dibujo ${ }^{26}$, agrega una quinta (que aparece en las banderas recordadas).

Así como en el Canto I del Infierno Dante usa al leone, la lonza y la loba (animales reales) con fines alegóricos, habría podido usar estrellas reales con el mismo fin, pero me parece forzar los argumentos de manera innoble. Con respecto a la primera gente, que habría visto esas cuatro estrellas, sería una alusión a Adán y Eva, que habitaron el Paraíso Terrestre, que ahora el poeta ha colocado en la cima de la montaña del Purgatorio. Y así lo creyeron Pietro Alighieri, Buti, el Anonimo Fiorentino y los comentadores modernos ${ }^{27}$. Sin embargo, no faltan otras interpretaciones: el Lana dice que son los hombres de la Edad de Saturno o del Oro; Benvenuto, citando a San Agustín (De civitate Dei, XV), afirma que se trata de los antiguos romanos que practicaron las virtudes ${ }^{28}$. Hay también varias que son discutibles y de dudosa validez científica ${ }^{29}$.

Para concluir, se reafirma la naturaleza simbólica de las estrellas con las mismas señales puestas en evidencia por Dante mismo. En efecto, dice de inmediato:

\section{Goder pareva il ciel di lor fiammelle. Oh settentrional vedovo sito,}

\footnotetext{
${ }^{25}$ Desde Ver ad locum. Para la referencia bibliográfica de Jacopo della Lana y Francesco BUTI, cfr. nota 3. Para Benvenuto, cfr. nota 4. Los otros comentarios son: L'Ottimo commento della Divina Commedia testo inedito d'un contemporaneo di Dante, publicado por Torri, Alessandro, Pisa: N. Capurro, 1827-29. y Commento alla Divina Commedia d'Anonimo Fiorentino del secolo XIV, publicado por Fanfani, Pietro, Bolona: Gaetano Romagnoli, 1866-74.

${ }^{26}$ Ver Lámina I.

27 Ver ad locum. Para no aumentar innecesariamente la mole de estas notas, obvío los nombres de los comentadores modernos. Para las restantes referencias del párrafo, me remito a las ediciones ya citadas.

${ }^{28}$ Ostendit Deus in opulentissimo regno Romanorum quantum civiles virtutes valeant etiam sine vera religione (Dios mostró en el poderosísimo reino de los Romanos cuánto valen las virtudes civiles incluso sin religión verdadera; ad locum.) En todo caso, la referencia de Benvenuto está equivocada, porque él mismo cita este texto en el Cap. V de su Romuleon como si formara parte del Libro XVIII del De civitate Dei, pero no corresponde al texto agustiniano).

${ }^{29}$ Por ejemplo, me resulta completamente fuera de lugar imaginar que esa primera gente pueda ser el pueblo de la Atlántida, el mítico continente que habría desaparecido en el 10.000 antes de Cristo. Así lo pretenden algunos especuladores anónimos, que figuran en Internet, y que no toman en cuenta que Dante no conoció la República de Platón (principal fuente literaria de esa tradición historiográfica).
} 
poiché privato se’ di veder quelle!

Com'io da loro sguardo fu'partito, un poco me volgendo a l'altro polo, là onde il Carro già era sparito,

vidi presso di me un veglio, solo, degno di tanta reverenza in vista, che più non dèe al padre alcun figliuolo.

Lunga la barba, di pel bianco mista, portav', ai suo capelli simigliante, de' quai cadev'al petto doppia lista.

Li raggi delle quattro luci sante fregiavan si la sua faccia di lume ch'i' 'l vedea come 'l sol fosse davante. (Pg I, 25-39).

[Gozar parecía el cielo con sus llamitas. ¡Oh, septentrional sitio viudo, porque privado estás de ver aquéllas! Cuando yo de ellas mi mirada aparté, volviéndome al otro polo, allá donde el Carro había ya desaparecido del otro polo, vi junto a mí a un viejo, solo, digno de tanta reverencia a la vista, que más no debe algún hijo al padre. Llevaba larga la barba, mixta de pelo blanco, semejante a sus cabellos, de los cuales caía al pecho doble lista. Los rayos de las cuatro luces santas frisaban de luz así su cara que yo lo veía como si el sol estuviese delante.]

El septentrional sitio viudo es claramente el hemisferio boreal que no puede ver esas estrellas (quizás por lo cual los hombres que allí habitan han seguido los vicios). El cielo austral, en cambio, goza de ellas y -precisamente- aparece un anciano que despierta la reverencia que un hijo debe a un padre. Se trata de Catón $y$-no por casualidad- los rayos de las estrellas iluminan su rostro como si fuese el sol.

La alegoría me parece transparente: está iluminado por la gracia divina, así como esas luces iluminan el camino de aquéllos que ascienden por la montaña del Purgatorio. 
Como si no bastara, en el Paraíso Terrestre, las cuatro virtudes aparecen personificadas en la forma de cuatro jovencitas que están al lado izquierdo del carro que representa a la Iglesia y que -después que Dante ha sido sumergido en el río Leteo - cantan:

Indi mi tolse, e bagnato m'offerse

dentro a la danza delle quattro belle;

e ciascuna del braccio mi coperse.

«Noi siam qui ninfe, e nel ciel siamo stelle;

pria che Beatrice discendesse al mondo,

fumo ordinate a lei per sue ancelle.

Merrenti a li occhi suoi; ma nel giocondo

lume ch'è dentro aguzzeranno i tuoi

le tre di là, che miran più profondo». (Pg XXXI, 103-111).

[Después me sacó, y mojado me ofreció dentro de la danza de las cuatro bellas; y cada una me cubrió con un brazo. "Nosotras aquí somos ninfas, y en el cielo somos estrellas; antes de que Beatriz descendiese al mundo, fuimos destinadas como esclavas suyas a ella. Te conduciremos ante sus ojos; pero en la alegre luz que está dentro agudizarán los tuyos las tres de allá, que miran más profundo»].

Las tres de allá son, obviamente, las tres virtudes teologales, que está a la derecha del carro. ¡Todo resulta absolutamente claro! Con todas estas indicaciones, resulta imposible que Dante mencionase las cuatro estrellas de la constelación del Centauro que, cercanas al polo antártico se identifican como la Cruz del Sur.

En el Antipurgatorio son los maitines, la última de las horas canónigas, antes del despuntar del alba. Ésta comienza a vencer a la obscuridad, iluminando el cielo y dando transparencia al aire. Al mismo tiempo, Dante advierte el movimiento ligero de las olas del mar.

L'alba vinceva l'ora mattutina

che fuggia innanzi, si che di lontano

conobbi il tremolar de la marina. (Pg I, 115-117). 
[El alba vencía a la hora de maitines que adelante huía, así como a lo lejos conocí el trémulo de la marina].

Ahora, las referencias horarias van a ser dictadas por la posición del sol. Por ejemplo, mientras Virgilio habla con Estacio y lo viajeros se encuentran en el $6^{\circ}$ círculo, destinado a los golosos:

e già le quattro ancelle eran del giorno rimase indietro, e la quinta era al tèmo, drizzando pur in sù l'ardente corno, (Pg XXII, 118-120).

[y ya las cuatro esclavas del día habían quedado atrás, y la quinta estaba al timón, dirigiendo no obstante hacia arriba el cuerno ardiente,]

Las primeras cuatro horas del día habían pasado y la quinta estaba al timón del carro del sol, dirigiendo hacia lo alto su punta llameante. Esto porque las horas eran las ministras del carro del sol, del cual guiaban el caballo (vedi che torna / dal servigio del dí l'ancella sesta - mira que la esclava sexta vuelve del servicio del día; Pg XII. 80-81). Vale decir, era ya una hora entre las diez y las once de la mañana del sábado 9 de abril.

\section{REFERENCIAS}

Alighieri, Dante. La Commedia, testo critico secondo i più antichi codici fiorentini a cura di Antonio Lanza. Anzio: De Rubeis, 1996, ad locum.

Alighieri, Pietro. Petri Allegherii super Dantis ipsius genitoris Comoedian commentarium, publicado por Vincenzo Nannucci. Firenze: Piatti, 1845.

Anónimo Fiorentino. Commento alla Divina Commedia d'Anonimo fiorentino del secolo XIV, publicado por Pietro Fanfani. Bologna: Gaetano Romagnoli, 1866-74. 3 vols.

Benvenuto da Imola. Benvenuti de Rambaldis de Imola Comentum super Dantis Aldigherii Comoediam, publicado por James Philip Lacaita. Firenze: Barbèra, 1887. 5 vols. 
Blanco Jiménez, José. "Bene ascolta chi la nota" (If. XV, 99) Intertextualidad clásica en la Commedia de Dante. Santiago de Chile: Ediciones Video Carta, 2007.

Buti, Francesco da. Commento di Francesco da Buti sopra la Divina Commedia di Dante Alighieri, publicado por Crescentino Giannini. Pisa: Nistri, 185862.3 vols.

Buti, Giovanni - Bertagni, Renzo. "Carro". Enciclopedia dantesca. Roma: Treccani, 1970, I, $851 b-852$ a.

Camilli, Amerindo. "La cronologia del viaggio dantesco", Studi Danteschi XXIX (1950): 61-84. Es sobre todo un fonetista (Servigliano, 1879-1960).

Curtius Rufus, Quintus. Curti Rufi Historiarum Alexandri Magni Macedonis libri qui supersunt, publicado por Theodor Vogel. Leipzig, Teubner: 1903-1906. 2 vols. Reimpreso en 2002.

Lana, Iacopo della. Comedia di Dante degli Alighieri col commento di Iacopo della Lana bolognese, publicado por Luciano Scarabelli. Bologna: Tipografia Regia, 1866-67. 3 vols.

Manuguerra, Mirco. "La fisica di Dante e l'enigma astronomico della datazione del viaggio nella Divina Commedia”. Atti del XVII Congresso Nazionale di Storia della Fisica e dell'Astronomia, C.N.R. - Consiglio Nazionale delle Ricerche, Commissione di Studio per la Storia della Fisica e dell'Astronomia, Como, Centro Volta - Villa Olmo, 23-24 maggio 1997.

Manuguerra, Mirco. "Una soluzione teologico-astronomica coerente per l'enigma della datazione del Viaggio nella Commedia", L’Alighieri XLIV, n. 21 (2003): 109-114. Es el presidente del Centro Lunigianese di Studi Danteschi, con sede en Mulazzo (Massa Carrara, Italia).

Moore, Edward. Gli accenni al tempo nella Divina Commedia e loro relazione con la presunta data e durata della visione, traducción italiana de Cino Chiarini. Roma: Salerno, 2007.

Polo, Marco. Il milione di Marco Polo: testo di lingua del secolo decimoterzo ora per la prima volta pubblicato ed illustrato dal conte Gio. Battista Baldelli-Boni. Firenze: da' torchi di G. Pagani, 1826. 
Ramusio, Giovan Battista. Navigationi et viaggi. Venezia: Lucantonio Giunti, $1550-1559.3$ vols.

Venturi, Pompeo. La Divina Commedia di Dante Alighieri...col comento del P. Pompeo Venturi. Lucca: Cappurri, 1732. 3 vols.

Vespucci, Amerigo. Lettere di viaggio, publicada por Luciano Formisano. Milano: Mondadori, 1985. 